\title{
Effect of quantum interference in the nonlinear conductance of microconstrictions
}

\author{
A. Namiranian ${ }^{1}$, Yu.A. Kolesnichenko ${ }^{1,2}$, A.N. Omelyanchouk ${ }^{2}$ \\ 1 Institute for Advanced Studies in Basic Sciences, Gava Zang, Zanjan 45195-159, Iran \\ 2 B. Verkin Institute for Low Temperature Physics and Engineering, \\ National Academy of Sciences of Ukraine, \\ 47 Lenin Ave., 310164 Kharkov, Ukraine
}

(November 21, 2018)

\begin{abstract}
The influence of the interference of electron waves, which were scattered by single impurities, on nonlinear mentally 4 ) is studied theoretically. The dependence of the interference pattern in the conductance $G(V)$ on the contact diameter and the spatial distribution of impurities is analyzed. It is shown that the amplitude of conductance oscillation is strongly depended on the position of impurities inside the constriction.
\end{abstract}

\section{INTRODUCTION.}

The Scanning Tunneling Microscopy (STM) and the Mechanically Controllable Break-junction (MCB) techniques offer an opportunity to study the conductance of metallic contacts consisting of only a few atoms (quantum contacts). The electrical conductance of such contacts, at small bias voltage is proportional to the number of propagating electron modes, $N$, each one contributing an amount of $G_{0}=2 e^{2} / h$ 3. With increasing the diameter of the contact the energies of modes continuously decreases, but the number of modes increases whenever a new mode fits into the constriction cross section. This number, $N$, is limited by the requirement that the kinetic energy for the transverse motion is smaller than the Fermi energy $\varepsilon_{F}$. When a new mode is occupied, a new quantum channel is opened. The conductance then undergoes a jump of $G_{0}$. Such quantization of conductance has been observed in both two and three dimensional contacts with diameters comparable to Fermi wave-length $\lambda_{F}=h / p_{F}\left(p_{F}\right.$ is the Fermi momentum $)$ - 8. Jumps in conductance are also expected to occur, at the constant contact diameter while bias voltage is varied. If the bias $e V$ is larger than the distances between the energy levels of quantum modes, it is possible to change the number of opened modes by changing the voltage $V$. At a certain threshold voltage a channel is opened or closed for one direction of the electron wave vector along the constriction and consequently conductance suffers a $G_{0} / 2$ stepwise change 9 .

Quantum interference effects have been studied in different mesoscopic systems 10 . In ballistic metallic microconstrictions it manifests itself as fluctuations in conductance when a magnetic field 11 or an electrical voltage is applied12. Now experimental efforts have been done using MCB techniques, in order to measure conductance as a function of voltage in atomic-size point contactst. A prominent feature of these measurements, is the existence of small random voltage-dependent fluctuations in conductance, far from steps. The measurements 1 clearly indicate suppression of the fluctuations for conductance values near the integer multiples of the conductance quantum. Similar results have been reported by using a STM to show the strong voltage dependence of conduftance of one-atom contacts at different temperaturest. It is generally believed that the observed oscillations in conductance are due to the quantum interference effects 2 . Ludoph and co-authorst, propose the following interpretation: The electron wave transmitted through the contact is backscattered to the contact by an impurity and then partially reflected at the contact. These waves interfere and change the total conductivity. The energy and thus the wave number of an injected electron into the channel, depends on the voltage. Consequently the interference pattern in conductance oscillates as electron wave number varies with the voltage.

Although the theory developed by Ludoph et al. 1 can explain the general feature of fluctuations but here we try to examine a different mechanism. Impurities (or defects) are assumed to be located inside the constriction, and the interference is effectively between waves scattered from the impurities. The existence of a few defects or impurities inside the constriction is rather natural considering the way the contact is formed. Using the model of a long microconstriction we can find the conductivity analytically. We discuss the theory of nonlinear electron transport through a mesoscopic microconstriction with a few impurities. We show that the nonlinear dependence of the quantum conductance on the voltage is obtained from this model. The form of this dependence is affected not only by the distances between impurities, but also by their positions inside the constriction.

In Sec. II the model Hamiltonian is discussed and is used to obtain a general expression for the nonlinear conductance. In Sec. III a $\delta$-function potential is assumed for the interaction of electrons with impurities and a simplified equation for the conductance is obtained. Within the framework of perturbation theory, a general analytical equation is also derived for conductance of the sys- 
tem, for arbitrary number of quantum modes and arbitrary number of impurities located in arbitrary positions. These analytical results are illustrated by numerical calculations for the contact in the form of a long cylindrical contact.A brief discussion of result is given in Sec. IV.

\section{GENERAL EQUATION FOR THE NONLINEAR CONDUCTANCE OF THE LONG QUANTUM MICROCONSTRICTION.}

Let us consider a long narrow constriction, which connects two bulk metals, assuming $e V \ll \varepsilon_{F}$. The geometry is shown in Fig. 1. We assume that the contact shape is smooth on the scale of the wavelength $\lambda_{F}$. This condition assures that different transverse modes pass through the ballistic contact independently (adiabatic approximation 13 ). We also assume that the contact length is much larger than its diameter and we can neglect the constriction end effects. Under these approximations, the electrical field inside the contact far from the ends is negligible and the energy $\varepsilon$ of ballistic electrons depends only on the sign of velocity along the contact axis 14.15. The Hamiltonian $H$ of the electrons contains the following terms:

$$
H=H_{0}+H_{1}+H_{i n t},
$$

where

$$
H_{0}=\sum_{n} \varepsilon_{\alpha} c_{\alpha}^{\dagger} c_{\alpha}
$$

is Hamiltonian of free electrons, and

$$
H_{1}=\frac{e V}{2} \sum_{\alpha} \operatorname{signv}_{z} c_{\alpha}^{\dagger} c_{\alpha}
$$

describes the influence of applied bias voltage. $H_{\text {int }}$ denotes interaction of electrons with impurities, and depends on the positions of impurities $\mathbf{r}_{i}$ in the constriction; the operator $c_{\alpha}^{+}\left(c_{\alpha}\right)$ creates (annihilates) a conduction electron with the wave function $\varphi_{\alpha}$, and energy $\varepsilon_{\alpha}$. The electron wave functions and eigenvalues are

$$
\begin{gathered}
\varphi_{\alpha}(\mathbf{r})=\psi_{\beta}(\mathbf{R}) \exp \left(\frac{i}{\hbar} p_{z} z\right) ; \\
\varepsilon_{\alpha}=\varepsilon_{\beta}+\frac{p_{z}^{2}}{2 m_{e}}
\end{gathered}
$$

where $\alpha=\left(\beta, p_{z}\right), \beta$ is the set of two transverse quantum numbers; $p_{z}$ is the momentum of an electron along the contact axis. $\mathbf{r}=(\mathbf{R}, z), \mathbf{R}$ is a coordinate in the plain, perpendicular to the $z$ axis $m_{e}$ is the electron mass.

In zero approximation in $H_{\text {int }}$ the current $J_{0}$ through the contact area $S_{c}$ is

$$
J_{0}=e S_{c} \operatorname{Tr}\left(v_{z} \rho_{0}\right)
$$

where

$$
\rho_{0}=f_{F}\left(H_{0}+H_{1}\right) ;
$$

$v_{z}=p_{z} / m$ is the electron velocity; $f_{F}$ is the Fermi function. Using the Eqs.(6),(7), and wavefunctions (4), we find the equation for the ballistic conductance:

$$
G_{1}=\frac{1}{2} G_{0} \sum_{\beta}\left[f_{F}\left(\varepsilon_{\beta}+\frac{e V}{2}\right)+f_{F}\left(\varepsilon_{\beta}-\frac{e V}{2}\right)\right]
$$

At zero temperature and $V \rightarrow 0$, this formula describes the well known $G_{0}$ steps of quantum conductance and in the quasiclassical case it turns into the Sharvin conductance 14.

In order to investigate the influence of single impurities on the nonlinear quantum conductance of the point contact, we use the method, which was developed Kulik and others 18 . 19 . The change in the electrical current $\Delta J$ is related to the rate of energy dissipation by the relation:

$$
\Delta J V=\frac{d E}{d t}=\frac{d\left\langle H_{1}\right\rangle}{d t}
$$

Differential of $\left\langle H_{1}\right\rangle$ with respect to time $t$ is we obtained from Heisenberg equation. The change $\Delta J$ of the current due to interactions of electrons with impurities; would then be

$$
\Delta J V=\frac{1}{i \hbar}\left\langle\left[H_{1}(t), H_{i n t}(t)\right]\right\rangle
$$

where

$$
\langle\ldots\rangle=\operatorname{Tr}(\rho(t) \ldots) .
$$

All operators are in the interaction representation.

The statistical operator $\rho(t)$ satisfies the equation

$$
i \hbar \frac{\partial \rho}{\partial t}=\left[H_{i n t}(t), \rho(t)\right]
$$

which can be solved using perturbation theory in $H_{\text {int }}$ (but for the arbitrary $H_{1}$ ):

$$
\rho(t)=\rho_{0}+\frac{1}{i \hbar} \int_{-\infty}^{t} d t^{\prime}\left[H_{i n t}\left(t^{\prime}\right), \rho_{0}\right]+\cdots
$$

We would then have

$$
\Delta J=-\frac{1}{\hbar^{2} V} \int_{-\infty}^{t} d t^{\prime} \operatorname{Tr}\left(\rho_{0}\left[\left[H_{1}, H_{i n t}(t)\right], H_{i n t}\left(t^{\prime}\right)\right]\right) \text {. }
$$

The decrease in total conductance $\Delta G=-G_{2}$, results the quantum interference is defined as

$$
G_{2}=-\frac{d \Delta J}{d V}
$$

If the applied bias $e V$ is much smaller than the differences between the energies $\varepsilon_{\beta}$ of modes, the Eq. (15) describes the dependence of total conductance on the voltage $V$. 


\section{THE CONDUCTANCE OSCILLATIONS.}

Now using the general Eqs. (14), (15), we investigate the behaviour of $G_{2}$ for the case of $\delta$ - function scattering potential. The Hamiltonian $H_{\text {int }}$ can be written as

$$
H_{\text {int }}\left(\mathbf{r}_{j}\right)=g \sum_{\alpha \neq \alpha^{*}} \varphi_{\alpha}^{*}\left(\mathbf{r}_{j}\right) \varphi_{\alpha^{\prime}}\left(\mathbf{r}_{j}\right) c_{\alpha}^{\dagger} c_{\alpha^{\prime}} .
$$

Here $g$ is the coupling constant of the interaction of an electron with an impurity located in the point $\mathbf{r}_{j}$.
Substituting Eqs.(7),(16) into Eq.(14), after some simple but cumbersome calculations we find

$$
\begin{gathered}
\Delta J=-\frac{e \pi}{2 \hbar} g^{2} \sum_{\alpha, \alpha^{\prime}} \sum_{i, j}\left(\operatorname{signv}_{z \alpha}-\operatorname{signv}_{z \alpha^{\prime}}\right) \times \\
\varphi_{\alpha^{\prime}}^{*}\left(\mathbf{r}_{j}\right) \varphi_{\alpha}^{*}\left(\mathbf{r}_{i}\right) \varphi_{\alpha^{\prime}}\left(\mathbf{r}_{i}\right) \varphi_{\alpha}\left(\mathbf{r}_{j}\right)\left(f_{\alpha^{\prime}}-f_{\alpha}\right) \delta\left(\epsilon_{\alpha^{\prime}}-\epsilon_{\alpha}\right),
\end{gathered}
$$

where $f_{\alpha}=f_{F}\left(\varepsilon+\frac{e V}{2} \operatorname{signv}_{z \alpha}\right)$. At zero temperature $f_{F}=\Theta\left(\varepsilon_{F}-\varepsilon\right)$, the Eq.(17) can be further simplified. Using the wave functions (4), we obtain for nonlinear part of conductance the following equation:

$$
\begin{gathered}
G_{2}=G_{0} \frac{\pi m_{e} g^{2}}{2} \sum_{\beta, \beta^{\prime}, i, j}\left\{\cos \left[\frac{1}{\hbar}\left(p_{\beta}^{(+)}+p_{\beta^{\prime}}^{(+)}\right) \Delta z_{i j}\right] \frac{1}{p_{\beta}^{(+)} p_{\beta^{\prime}}^{(+)}} \Theta\left(\varepsilon_{F}-\varepsilon_{\beta}+\frac{e V}{2}\right) \times\right. \\
\Theta\left(\varepsilon_{F}-\varepsilon_{\beta^{\prime}}+\frac{e V}{2}\right)+\cos \left[\frac{1}{\hbar}\left(p_{\beta}^{(-)}+p_{\beta^{\prime}}^{(-)}\right) \Delta z_{i j}\right] \frac{1}{p_{\beta}^{(-)} p_{\beta^{\prime}}^{(-)}} \Theta\left(\varepsilon_{F}-\varepsilon_{\beta}-\frac{e V}{2}\right) \times \\
\left.\Theta\left(\varepsilon_{F}-\varepsilon_{\beta n^{\prime}}-\frac{e V}{2}\right)\right\} \psi_{\beta^{\prime}}^{*}\left(\mathbf{R}_{j}\right) \psi_{\beta}^{*}\left(\mathbf{R}_{i}\right) \psi_{\beta^{\prime}}\left(\mathbf{R}_{i}\right) \psi_{\beta}\left(\mathbf{R}_{j}\right) .
\end{gathered}
$$

Where

$$
p_{\beta}^{( \pm)}=\sqrt{2 m_{e}\left(\varepsilon_{F} \pm \frac{e V}{2}-\varepsilon_{\beta}\right)} .
$$

The cosine terms in the Eq. (18) describe the conductance oscillations due to the interference of electrons waves scattered by impurities. The transverse parts $\psi_{\beta}\left(\mathbf{R}_{j}\right)$ of wave functions contain the mesoscopic effect of impurity positions inside the constriction. The equation (18) diverges at $p_{\beta}^{( \pm)}=0$. Physically it means that in the Born approximation the slowly moving electron is repeatedly scattered on the impurity. In this case the perturbation theory (Born approximation) is not valid any more, and we must take into account the interference of partial waves under the electron scattering by impurity. We assume that energy levels are not very close to the boundary energies $\varepsilon_{F} \pm \frac{e V}{2}$ and the quantity $G_{2}$ added to the ballistic conductance $G_{1}(8)$ is small.

For the numerical calculations we have used the free electron model of point contact consisting of two infinite half-spaces connected by a long ballistic cylinder of a radius $R$ and a length $L$ (Fig.1).

In the limit $L \rightarrow \infty$ the electron wave functions $\varphi_{\alpha^{\prime}}(\mathbf{r})$ and energies $\varepsilon_{\alpha}$ can be written as

$\varphi_{\alpha^{\prime}}(\mathbf{r})=\frac{1}{\sqrt{\Omega} J_{m+1}\left(\gamma_{m n}\right)} J_{m}\left(\gamma_{m n} \frac{\rho}{R}\right) \exp \left(i m \varphi+\frac{i}{\hbar} p_{z} z\right) ;$

where

$$
\varepsilon_{\alpha}=\varepsilon_{m n}+\frac{p_{z}^{2}}{2 m_{e}} ; \quad \varepsilon_{m n}=\frac{\hbar^{2}}{2 m_{e} R^{2}} \gamma_{m n}^{2}
$$

We have used cylindrical coordinates $\mathbf{r}=(\rho, \varphi, z)$ with $z$ along the axis of cylinder. Here $a=\left(n, m, p_{z}\right)$ are the quantum numbers, $\Omega=\pi R^{2} L$ is the volume of the channel, $\gamma_{m n}$ are the n-th zero of the Bessel function $J_{m}$. Since the electron energy has degeneracy for azimuthal quantum number $m$ ( as a result of the symmetry of the model), quantum modes with $\pm m$ have the same contribution in conductance. In this model, Sharvin cenductance has not only steps $G_{0}$, but also steps $2 G_{0} 17$. In Fig. 2 the dependence of the interference pattern on the number of impurities inside a constriction with constant radius is shown. It shows that as a result of the interference of electron waves, which were scattered by different impurities, the interference maxima in $G_{2}(V)$ dependence, may both be depressed and increased. The interference oscillation of the conductance depends strongly on the number of opened quantum modes which follows from the dependence of its maximum value of longitudinal electron momentum (see, Eq. 3) on the contact size. The voltage dependence of $G_{2}$ for different contact sizes are shown in Fig.3. Fig. 4. illustrates how the changing in the nonlinear dependence $G_{2}(V)$ changes with the contact size. It corresponds to the case of two impurities in the contact. The position of impurities and the number of opened quantum modes are kept constant. The difference in the interference oscillations is a result of the changing in the relative positions of nodes and maxima of the electron wave function from the points, in which the impurities are situated.

\section{CONCLUSION}

The dependence of quantum conductance of metallic ultrasmall contacts containing impurities on bias voltage has been theoretically studied. We have shown that impurities situated inside the quantum microconstriction produce a nonlinear dependence of the conductance on the applied voltage, which is the result of the interference 
of electron waves reflected by impurities. The transmission probability of the electron through constriction depends on the relation between the electron wave length $\lambda$ and $\Delta z_{i j}$, the projection of distances between impurities along the channel. It is maximum when the condition $\Delta z_{i j}=\frac{n \lambda}{2}$ ( $n$ is integer) is satisfied. Since the electron momentum depends on the applied bias, one can change the transmission by changing the voltage. Our numerical calculations show that the resulting nonmonotonic dependence of the conductance, is similar in shape to the ones observed in experiment, 2. The amplitude of interference pattern is sensitive to the transversal position of impurities inside the constriction. If the impurity is located near the point, where the electron wave function corresponding to the $n$th quantum mode vanishes, then the decreasing of transmission of that mode would be negligible.

We acknowledge fruitful discussion with M.R.H. Khajehpour and I.K.Yanson.

${ }^{1}$ B.Ludoph, M.H. Devoret, D.Esteve, C.Urbina and J.M. van Ruitenbeek, Phys. Rev. Lett. 82, 1530 (1999).

${ }^{2}$ C. Untidt, G. Rubo Bollinger, S. Vieira, N. Agrait,In Quntum and Mesoscopic Phenomena and Mesoscopic Devices in Microelectronics (Bilkent University, Ankara, Turkey, 1999) pp 78.

${ }^{3}$ For review, see Solid State Physics 44, Edit. H. Ehrenreich and Turnbull (Academic Press, New York, 1991) pp 1-228.

${ }^{4}$ B.J. van Wees, H. van Houten, C.W.J. Beenakker, J.G. Williamson, L.P. Kouwenhoven, D. van der Marel and C.T. Foxon, Phys. Rev. Lett. 60, 848 (1988).

${ }^{5}$ D.A. Wharam, M. Pepper, H. Ahmed, J.E.F. Frost, D.G. Hasko, D.C. Peacock, D.A. Ritchie, G.A.C. Jones, J. Phys. C: Solid State Phys. 21, L209 (1988).

${ }^{6}$ J.I. Pascual, J. Mendez, J.Gomez-Herrero, A.M. Baro, N.Garsia, Vu Thien Binh, Phys. Rev. Lett. 71, 1852 (1993).

${ }^{7}$ N. Agrait, J.G. Rodrigo, S. Viera, Phys. Rev. B 47, 12345 (1993).

${ }^{8}$ C.J. Muller, J.M. van Ruitenbeek, L.J. de Jongh, Phys. Rev. Lett. 69, 140 (1992).

${ }^{9}$ A.M. Zagoskin, JETP Lett., 52, 435 (1990).

${ }^{10} \mathrm{Y}$. Imry, Introduction to Mesoscopic Physics (Oxford, New York, 1997).

${ }^{11}$ P.A. Lee, A.D. Stone, Phys. Rev. Lett. 55, 1622 (1985).

12 P.A.M. Holweg, J.A. Kokkedee, J. Caro, A.H. Verbruggen, S. Radelaar, A.G.M. Jansen, and P.Wyder, Phys. Rev. Lett. 67, 2549 (1991).

${ }^{13}$ L.I. Glazman, G.B. Lesovik, D.E. Khmel'nitskii, R.I. Shekhter, JETP Lett. 48, 238 (1988).

${ }^{14}$ I.O. Kulik, A.N. Omelyanchouk, R.I. Shekhter, Sov. J. Low Temp. Phys. 7, 740 (1977).

${ }^{15}$ I.O. Kulik, I.K. Yanson, Sov. J. Low Temp. Phys., 4,596 (1978).

16 A. Szafer, A.D. Stone, Phys. Rev. Lett. 62, 300 (1989).
${ }^{17}$ E.N.Bogachek, A.M. Zagoskin, I.O. Kulik, Sov. J. Low Temp. Phys. 16, 796 (1990).

${ }^{18}$ I.O.Kulik, JETP, Pis'ma, 5, 423 (1967).

${ }^{19}$ I.O. Kulik, A.N. Omelyanchouk, I.G.Tuluzov, Sov. J. Low Temp. Phys., 14, 149 (1988).

${ }^{20}$ Yu. V. Sharvin, Sov. Phys. JETP, 21, 655 (1965). 


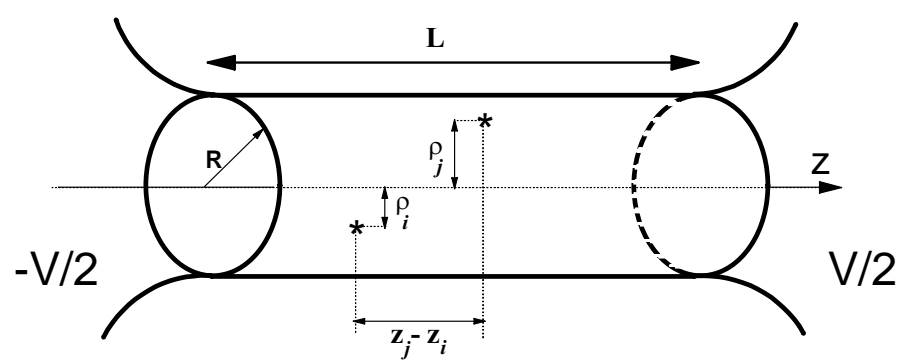

FIG. 1. The model of the nuantum mirroennctriction in the form of a lono rhannel of the radius $R$, which smoothly (on the Fermi length s schematically.

$\mathbf{G}_{2} / \mathbf{G}_{1}$

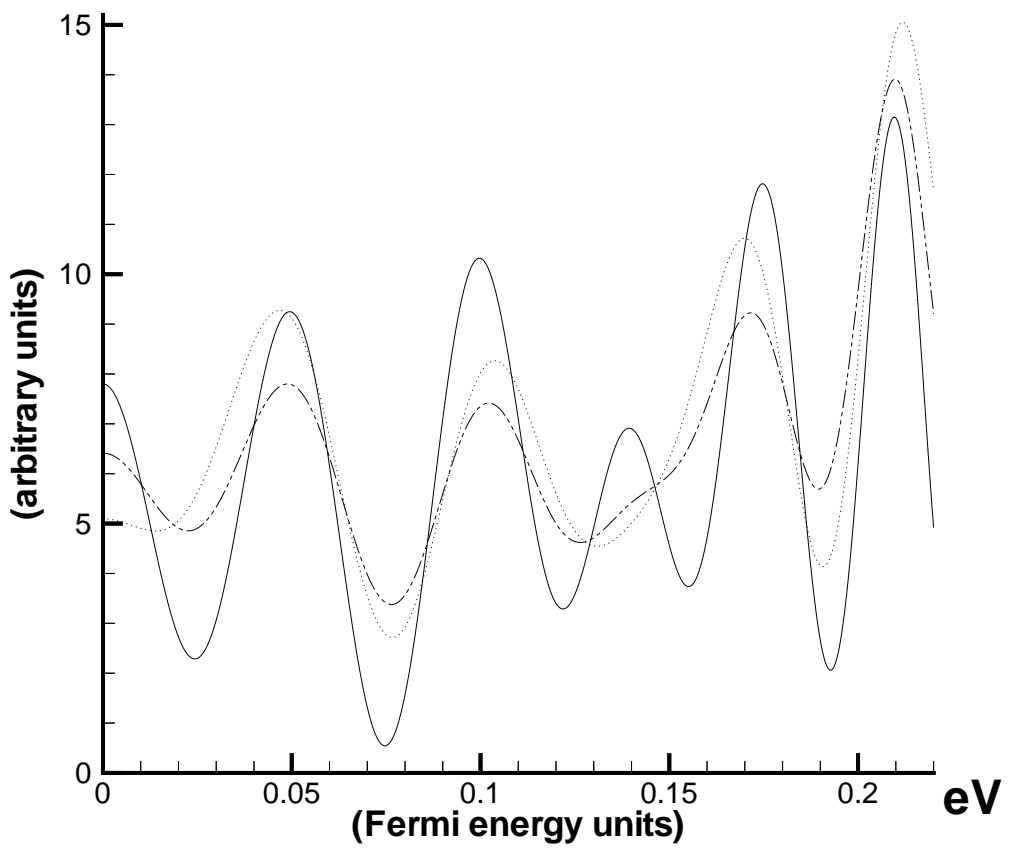
onstriction are shown

FIG. 2. The normalized conductance $G_{2} / G_{1}$ as a function of voltage in a three modes channel $\left(2 \pi R=4.2 \lambda_{F},\right)$ for the different number $k$ of impurities; $k=2$ for solid line, $k=3$ for dashed line and $k=4$ for dotted line. 


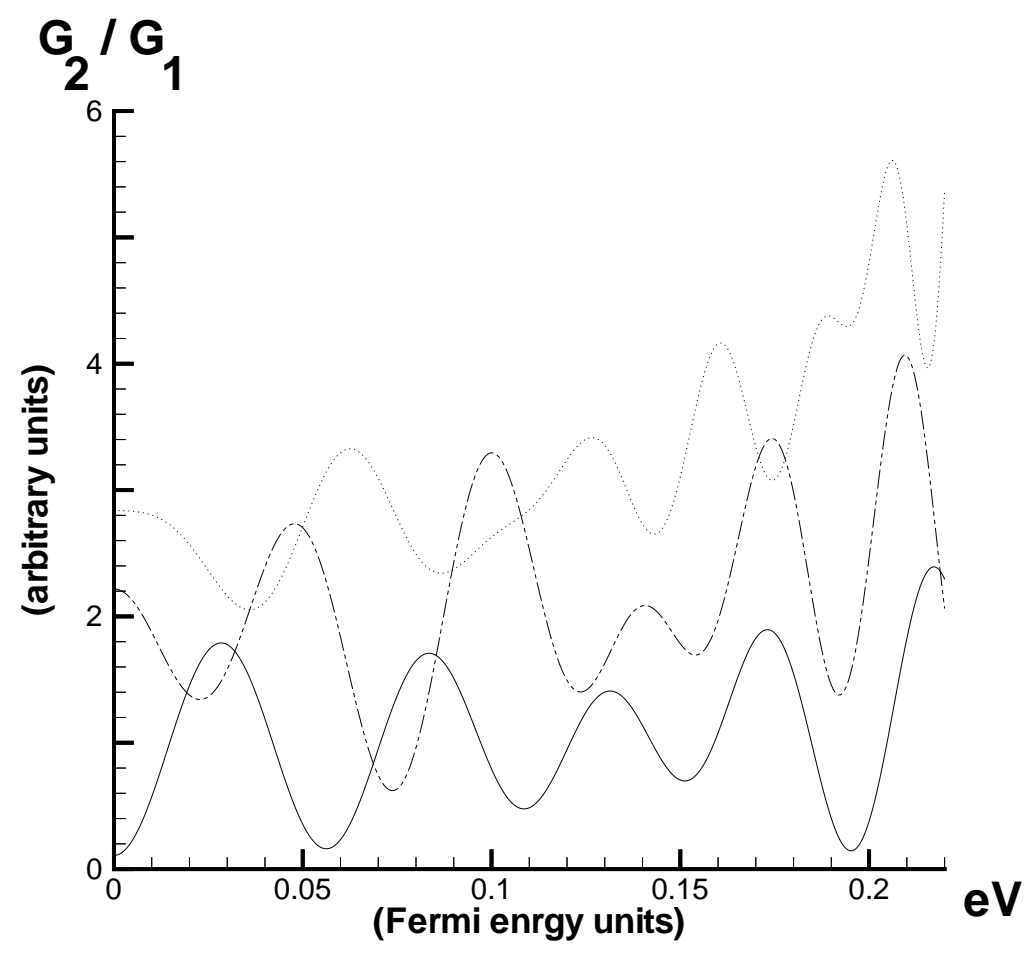

FIG. 3. The normalized conductance $G_{2} / G_{1}$ as a function of voltage for a channel with two impurities at different number of opened quantum modes; single mode $\left(2 \pi R=2.7 \lambda_{F}\right)$, for solid line, three modes $\left(2 \pi R=4.2 \lambda_{F}\right)$, for dashed line and five modes $\left(2 \pi R=5.5 \lambda_{F}\right)$ for dotted line.

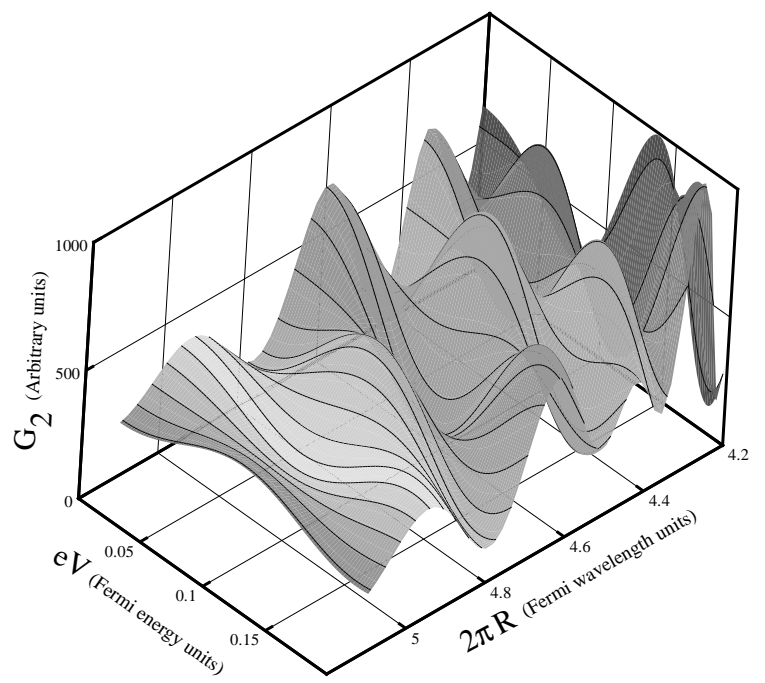

FIG. 4. The changing of the interference pattern in the $G_{2}(V)$ dependence of three mode channel, which contains two impurities, with increasing the contact diameter. The distance between impurities and its distances from the contact axis is the same for all values of $R$. The radius $R$ is changed in the interval, in which the number of opened quantum modes is constant. 